\title{
Nonlinear polarizers based on four-wave mixing in high-birefringence optical fibers
}

\author{
Massimiliano Guasoni* and Stefan Wabnitz \\ Dipartimento di Ingegneria dell'Informazione, Università di Brescia, Via Branze 38, 25123 Brescia, Italy \\ ${ }^{*}$ Corresponding author: massimiliano.guasoni@ing.unibs.it
}

Received January 3, 2012; revised March 8, 2012; accepted March 19, 2012;

posted March 21, 2012 (Doc. ID 160744); published June 1, 2012

Parametric amplification or four-wave mixing in high-birefringence optical fibers may be exploited to implement a novel type of nonlinear polarizer. Such a device leads to the simultaneous amplification, frequency conversion, and repolarization of both signal and idler waves along one of the principal birefringence axes of the fiber, independently of the pump, signal, and idler input state of polarization, power, and frequency detuning. We discuss the conditions for the observation of polarization attraction in fiber optics parametric amplifiers operating with a pump in either the normal or the anomalous dispersion regime. (C) 2012 Optical Society of America

OCIS codes: $\quad 230.5440,060.4370,230.1150,230.4320$.

\section{INTRODUCTION}

Optical devices that enable the dynamic control of the state of polarization (SOP) of light are key components in today's lightwave communication networks. Indeed, it is well known that the output SOP of a signal from an optical fiber link exhibits random time fluctuations due to environmental, temperature, or mechanical variations, so that relatively fast SOP rotation speeds on the Poincaré sphere are observed [1]. Whenever optical signals from a telecommunication link are used in combination with polarization-sensitive components such as coherent receivers, optical switches, polarization demultiplexers, or sensors, it is thus necessary to stabilize the SOP at the fiber output. Conventional SOP control devices with relatively high polarization tracking speed are based on lithium-niobate electro-optical polarization transformers [2,3]. An entirely new class of fiber-based and potentially ultrafast SOP controllers has been introduced in recent years, based on third-order nonlinear polarization interactions among different waves within the optical fiber itself. Known all-optical SOP controllers (or nonlinear polarizers) come in two flavors. Consider first dissipative polarizers, where the polarization asymmetry of stimulated (e.g., Brillouin [4] or Raman [5,6]) scattering gain due to a fully polarized and relatively intense pump wave is exploited for the control of the signal SOP. For such devices, whenever fibers with suitably low birefringence or polarization mode-dispersion (PMD) are employed, a signal gets simultaneously amplified and repolarized at the fiber output. Besides the gain provided by the pump via the stimulated scattering process, nonlinear dissipative polarizers act as conventional linear polarizers; namely input signal SOP fluctuations result in signal intensity fluctuations [or relative intensity noise (RIN)] at the fiber output. On the other hand, conservative polarizers exploit the cross-polarization interaction between a signal and a pump wave of different frequency [7], or a pump with the same frequency but counterpropagating in the opposite direction [8,9]. These peculiar polarizers are also known as nonlinear lossless polarizers: no power exchange occurs between the signal and the pump; hence input signal SOP fluctuations no longer transform in output signal intensity fluctuations. Conservative or lossless polarizers have been experimentally demonstrated using either isotropic (i.e., perfectly circular) $[10,11]$ or randomly birefringent, lowPMD optical fibers [12]. In this work, we propose and analyze a different type of nonlinear polarizer that belongs to the class of dissipative polarizers, which is based on degenerate four-wave mixing (FWM) between the arbitrarily polarized pump, the Stokes signal, and the anti-Stokes idler waves in high-birefringence (hibi) optical fibers. Important applications of FWM in optical fibers include the phase-sensitive parametric amplification of a signal, as well as the frequency conversion of a signal into an idler wave. FWM is generally studied in the simple case of a scalar interaction between the three interacting waves. Just as it occurs for fiber Raman amplifers, polarizationindependence of the gain of practical fiber-optic parametric amplifiers (FOPAs) is generally required, which can be obtained by using two orthogonally polarized pump waves. In the case of a single polarized pump, it is well known that different phase-matching mechanisms exist for the FWM process in either low [13] or high [14] birefringence optical fibers, with a pump propagating in the normal or in the anomalous dispersion regime. Whenever phase matching (PM) is absent at low pump powers, but phase-matched exponential growth of sidebands is achieved at relatively high powers, the FWM process is more commonly referred to as modulation instability (MI). Although vector FWM and MI in birefringent fibers have been experimentally observed for a long time [14-16], to our knowledge the main attention was paid to the gain spectrum of the generated sidebands. The SOP of the parametrically amplified sidebands was earlier investigated by Freitas et al. [17] in the special case of a linearly polarized pump propagating in the anomalous dispersion regime of a low-PMD fiber. The resulting scalar MI process is responsible for the observed attraction of the sideband SOP towards the pump at the fiber output. 
In this work, restricting for simplicity our attention to the case of a hibi fiber, we performed a systematic study of the output polarization properties of both Stokes signal and anti-Stokes idler waves as a function of their frequency detuning and their input SOP, as well as of the pump SOP and power. In general, the SOP of the parametric sidebands periodically evolves along the fiber, so that both the orientation of the polarization ellipse as well as its degree of ellipticity significantly change along the propagation direction. Nevertheless, our study revealed the unexpected result that, whenever the pump power is below a certain critical value, the SOP of both signal and idler waves is quasi-linearly polarized, with its orientation aligned with one of the two birefringence axes of the fiber. That is, the output SOP of the sidebands remains close to a linear polarization state, independently of the input sideband SOP, their frequency detuning from the pump, as well as the pump SOP and power. We may refer to such an effect as pump-independent quasi-linear polarization attraction (QLPA) towards the principal birefringence axes of the fiber. In other words, the FOPA simultaneously acts as an amplifier and a polarizer for both idler and signal waves. Since the FOPA-based polarizer is a dissipative polarizer, unlike lossless polarizers, it is not immune from output RIN resulting from input polarization fluctuations. On the other hand, parametric gain is generally larger than Raman gain in silica fibers; therefore one may use shorter fibers or lower pump powers than with Raman polarizers. Moreover, in comparison with Raman gain-based polarizers, the use of parametric gain may lead to more flexibile devices since it is possible to tune the position of the amplified and repolarized sidebands by varying the fiber birefringence and the pump power.

Our paper is organized as follows: in Sections $\underline{2}$ and $\underline{3}$, we introduce the properties of the general solutions of the nonlinear coupled equations describing the FWM (or MI) process in a hibi fiber, and explicitly point out four different four-photon energyexchange processes that may be responsible for the QLPA effect; in Section 4 we describe, by means of numerical simulations, specific situations where the QLPA property is observed; and in Section 5 we present our conclusions.

\section{GENERAL THEORY}

Let us consider the interaction between a pump $(p)$, a signal $(s)$, and an idler (i) wave, that copropagate in the $z$ direction in a hibi fiber. The $x$ and $y$ components of the electric field envelope at the carrier frequency $\nu_{p}$ obey the following incoherently coupled nonlinear Schrödinger equations (CNLSE), where coherent nonlinear polarization coupling terms are neglected owing to the hibi hypothesis (see [16])

$$
\begin{aligned}
& \frac{\partial A_{x}}{\partial z}+\frac{1}{v_{x p}} \frac{\partial A_{x}}{\partial t}+\frac{\beta_{2}}{2} \frac{\partial^{2} A_{x}}{\partial t^{2}}=i \gamma\left(\left|A_{x}\right|^{2} A_{x}+\frac{2}{3}\left|A_{y}\right|^{2} A_{x}\right), \\
& \frac{\partial A_{y}}{\partial z}+\frac{1}{v_{y p}} \frac{\partial A_{y}}{\partial t}+\frac{\beta_{2}}{2} \frac{\partial^{2} A_{y}}{\partial t^{2}}=i \gamma\left(\left|A_{y}\right|^{2} A_{y}+\frac{2}{3}\left|A_{x}\right|^{2} A_{y}\right) .
\end{aligned}
$$

In Eq. (1), $v_{x p}$ and $v_{y p}$ are the group velocities for $x$ and $y$-polarized waves at angular frequency $\omega_{p}=2 \pi \nu_{p} ; \gamma=$ $n_{2} \omega_{p} /\left(c_{0} A_{\text {eff }, p}\right)$ is the nonlinear fiber coefficient, where $n_{2}=$ $3.2 \cdot 10^{-16} \mathrm{~cm}^{2} / \mathrm{W}$ is the nonlinear index, $c_{0}$ is the speed of light in vacuum, and $A_{\mathrm{eff}, p}$ is the effective area of the fundamental fiber mode; $\beta_{2}$ is the group velocity dispersion coefficient (supposed to be common for both polarizations), defined as

$$
\beta_{2}=\left.\frac{\partial^{2} k_{j p}}{\partial \omega^{2}}\right|_{\omega=\omega_{p}}, \quad(j=\{x, y\}),
$$

where $k_{j p}$ are the propagation constants of orthogonal fiber modes at the pump frequency.

In Eq. (1) we may decompose the field in a sum of pump, signal, and idler for each polarization as

$$
\begin{aligned}
A_{j}(z, t)= & A_{j p}(z)+A_{j s}(z) \exp \left(i \Delta k_{j s} z+i 2 \pi \Delta \nu t\right) \\
& +A_{j i}(z) \exp \left(i \Delta k_{j i} z-i 2 \pi \Delta \nu t\right)(j=\{x, y\}),
\end{aligned}
$$

where $\Delta \nu=\nu_{s}-\nu_{p}=\nu_{p}-\nu_{i}$ is the frequency detuning between pump and sidebands, $\Delta k_{j(s, i)}=k_{j(s, i)}-k_{j p}$, being $k_{j(s, i, p)}$ the propagation constants at the signal, idler, and pump frequencies, respectively. In the hypothesis of a $\mathrm{CW}$ undepleted pump, the pump $x$ and $y$ amplitudes are time-independent, and their evolution along the fiber is given by (see [16])

$$
\begin{aligned}
& A_{x p}(z)=\sqrt{P} \exp \left[i \phi_{x p}(z)\right], \\
& A_{y p}(z)=\sqrt{Q} \exp \left[i \phi_{y p}(z)\right], \\
& \phi_{x p}(z)=\psi_{x p}(0)+\gamma(P+(2 / 3) Q) z, \\
& \phi_{y p}(z)=\psi_{y p}(0)+\gamma(Q+(2 / 3) P) z,
\end{aligned}
$$

where $P$ and $Q$ are the pump powers along the $x$ and $y$ axes ( $P_{\text {tot }}=P+Q$ is the conserved total pump power), while $\psi_{x p}(0)$ and $\psi_{y p}(0)$ are the two input phases of the $x$ and $y$ pump components. Inserting Eq. (3) in Eq. (1), a set of ordinary differential equations governing sidebands propagation is obtained as

$$
\begin{aligned}
\frac{d A_{x s}}{d z} & =i \gamma\left(2\left|A_{x p}\right|^{2}+\frac{2}{3}\left|A_{y p}\right|^{2}\right) A_{x s} \\
& +i \gamma \frac{2}{3} \overbrace{A_{x p}^{2} A_{x i}^{*} \exp \left[i\left(2 k_{x p}-k_{x i}-k_{x s}\right)\right.}^{X_{1}}+i \gamma \frac{2}{3} A_{x p} A_{y p}^{*} A_{y s} \\
& +i \gamma \frac{2}{3} \overbrace{A_{x p} A_{y p} A_{y i}^{*} \exp \left[i\left(k_{x p}-k_{x s}-k_{y i}+k_{y p}\right)\right]}^{X_{3}}, \\
\frac{d A_{x i}}{d z}= & i \gamma \frac{2}{3} \overbrace{A_{x p}^{2} A_{x s}^{*} \exp \left[i\left(2 k_{x p}-k_{x i}-k_{x s}\right)\right]}^{X_{1}} \\
& +i \gamma\left(2\left|A_{x p}\right|^{2}+\frac{2}{3}\left|A_{y p}\right|^{2}\right) A_{x i} \\
& +i \gamma \frac{2}{3} \overbrace{A_{x p} A_{y p} A_{y s}^{*} \exp \left[i\left(k_{x p}-k_{x i}-k_{y s}+k_{y p}\right)\right]}^{X_{4}}+i \gamma \frac{2}{3} A_{x p} A_{y p}^{*} A_{y i}, \\
\frac{d A_{y s}}{d z} & =i \gamma\left(2\left|A_{y p}\right|^{2}+\frac{2}{3}\left|A_{x p}\right|^{2}\right) A_{y s} \\
& +i \gamma \frac{2}{3} \overbrace{A_{y p}^{2} A_{y i}^{*} \exp \left[i\left(2 k_{y p}-k_{y i}-k_{y s}\right)\right]}^{X_{2}}+i \gamma \frac{2}{3} A_{y p} A_{x p}^{*} A_{x s} \\
& +i \gamma \frac{2}{3} \overbrace{A_{x p} A_{y p} A_{x s}^{*} \exp \left[i\left(k_{y p}-k_{y i}-k_{x s}+k_{x p}\right)\right]}^{A_{y p} A_{x p}^{*} A_{x i} \cdot} \\
& +i \gamma \frac{2}{3} \overbrace{A_{y p} A_{x p} A_{x i}^{*} \exp \left[i\left(k_{x p}-k_{y s}-k_{x i}+k_{y p}\right)\right]}^{X_{3}}, \\
\frac{d A_{y i}}{d z}= & i \gamma \frac{2}{3} \overbrace{A_{y p}^{2} A_{y s}^{*} \exp \left[i\left(2 k_{y p}-k_{y i}-k_{y s}\right)\right]}^{\left.X_{2}+\frac{2}{3}\left|A_{x p}\right|^{2}\right) A_{y i}} \\
& +i(5)
\end{aligned}
$$


In the above equations, we may approximate $k_{y p}=$ $k_{x p}-2 \pi \nu_{p} \Delta W c_{0}$, where $\Delta n=n_{x}-n_{y}$ is the difference of effective refractive indexes of the two linearly polarized modes aligned with the linear birefringence axes; we set $n_{x}>n_{y}$ so that the $x(y)$ axis is the slow (fast) axis. We express the propagation constants of the signal and of the idler by means of a Taylor series development truncated at the second order; i.e., $k_{j s}=k_{j p}+v_{j p}^{-1} \Delta \omega+(1 / 2) \beta_{2} \Delta \omega^{2}$ and $k_{j i}=k_{j p}-v_{j p}^{-1} \Delta \omega+$ $(1 / 2) \beta_{2} \Delta \omega^{2}((j=\{x, y\}))$, being $\Delta \omega=2 \pi \Delta \nu$.

Labels $X_{(1,2,3,4)}$ in Eq. (5) indicate all possible four-photon energy-exchange processes involving the conversion of two pump photons in two sideband photons. Each of these processes may lead to sideband amplification, and it is characterized by an optimal sideband frequency detuning corresponding to peak sideband gain, i.e., the value $\Delta \nu_{(X 1, X 2, X 3, X 4)}$, which leads to the nonlinear PM of a specific FWM process. It is interesting to separately consider the four FWM processes, in order to analyze the conditions under which their corresponding PM frequencies are well separated. In this case, nonoverlapping FWM gain frequency bands may be obtained, each corresponding to a different FWM process, which permits a significant simplification of our analysis.

Let us start from the $X_{1}$ process: its PM condition reads as $\beta_{2}(2 \pi \Delta \nu)^{2}+2 \gamma P=0$, which can only be reached in the anomalous dispersion region $\beta_{2}<0$. The corresponding PM frequency is $\Delta \nu_{X 1}=(2 \pi)^{-1} \sqrt{2 \gamma P /\left|\beta_{2}\right|}$. In this process, which corresponds to scalar MI for $x$-polarized waves, two $x$-pump photons are converted into an idler $x$-photon and a signal $x$ photon: we may represent the process by using the notation $\left(x_{p}, x_{p}\right) \rightarrow\left(x_{s}, x_{i}\right)$. Similarly, in the $y$-polarized Michigan process $X_{2}$, the PM frequency is $\Delta \nu_{X 2}=(2 \pi)^{-1} \sqrt{2 \gamma Q / \beta_{2} \mid}$ and the four-photon energy-exchange may be represented as $\left(y_{p}, y_{p}\right) \rightarrow\left(y_{s}, y_{i}\right)$

The gain bands associated with $X_{1}$ and $X_{2}$ have peak gain values at $\Delta \nu_{X 1}$ and $\Delta \nu_{X 2}$, respectively. Although the two gain bands overlap, if $P>Q$, then $X_{1}$ leads to a larger MI gain than $X_{2}$ : clearly the number of conversions $\left(x_{p}, x_{p}\right) \rightarrow\left(x_{s}, x_{i}\right)$ is larger than the number of conversions $\left(y_{p}, y_{p}\right) \rightarrow\left(y_{s}, y_{i}\right)$. Therefore one may intuitively expect that for a sufficiently long fiber, a strong sideband attraction towards the $x$-axis is observed in a frequency gain band around $\Delta \nu_{X 1}$, the strength of this attraction being proportional to $P$. On the other hand, whenever $P<Q$, then $X_{2}$ is the dominating process, and the sidebands are attracted towards the $y$-axis in a frequency band around $\Delta \nu_{X 2}$, the strength of the corresponding attraction being proportional to $Q$. As far as $X_{3}$ is concerned, the corresponding PM condition reads as $\beta_{2}(2 \pi \Delta \nu)^{2}+(\Delta W c)(2 \pi \Delta \nu)+\gamma P_{\text {tot }}=0$, which can only be satisfied in the anomalous dispersion regime. It is important to note that PM does not depend on the input pump SOP but only on $P_{\text {tot }}$. Therefore, if we represent the energy-exchange process $X_{3}$ in terms of its four-photon conversion $\left(x_{p}, y_{p}\right) \rightarrow\left(x_{s}, y_{i}\right)$, strong attraction of the signal towards the $x$-axis and of the idler towards the $y$-axis is expected in a frequency band around $\Delta \nu_{X 3}$, independently of the input pump SOP. Nevertheless, the strength of the attraction is strongly dependent on the pump SOP since the $X_{3}$ gain is proportional to $\min (P, Q)$, i.e., to the number of photons pairs $\left(x_{p}, y_{p}\right)$ that can be converted by this process. Indeed, the FWM gain has a maximum whenever $P=Q$, and it vanishes if $P=0$ or $Q=0$.
Whenever the inequality

$$
P_{\text {tot }}<P_{c}=\frac{\Delta n^{2}}{4 \gamma c^{2} \beta_{2}}
$$

is satisfied, the PM frequency can be well approximated by $\Delta \nu_{X 3}=(2 \pi)^{-1}\left(\Delta W\left(c\left|\beta_{2}\right|\right)+\gamma P_{\text {tot }} c \Delta n\right)$. This means that the PM frequency can be linearly tuned by varying $P_{\text {tot }}$ around $(2 \pi)^{-1} \Delta w\left(c\left|\beta_{2}\right|\right)$, which represents the PM frequency in the low-power limit case. It is straightforward to show that if $P_{\text {tot }}<P_{c}$, then $\Delta \nu_{(X 1, X 2)}<(1 / 2) \Delta \nu_{(X 3)}$; therefore the FWM processes $X_{(1,2)}$ and $X_{3}$ are dominant in distinct frequency bands. To the contrary, whenever $P_{\text {tot }}>P_{c}$, the processes $X_{(1,2)}$ and $X_{3}$ compete in the same frequency band, so that a well-defined sideband attraction towards the birefringence axes as it occurs for each separate process cannot be expected any longer.

Finally, the PM condition for the $X_{4}$ process reads as $\beta_{2}(2 \pi \Delta \nu)^{2}-(\Delta N c)(2 \pi \Delta \nu)+\gamma P_{\text {tot }}=0$, which again is independent of the input pump SOP. However, differently from the previous cases, PM can only be reached in the normal dispersion regime (i.e., $\beta_{2}>0$ ). In this case, the energy-exchange process is $\left(x_{p}, y_{p}\right) \rightarrow\left(y_{s}, x_{i}\right)$; therefore a strong attraction of the idler towards the $x$-axis and of the signal towards the $y$-axis is expected in a frequency gain band around $\Delta \nu_{X 4}$, independently of the input pump SOP. Again under the condition $P_{\text {tot }}<P_{c}$, the PM frequency can be approximated as $\Delta \nu_{X 4}=(2 \pi)^{-1}\left(\Delta W\left(c\left|\beta_{2}\right|\right)-\gamma P_{\text {tot }} c / \Delta n\right)$. On the other hand, whenever $P_{\text {tot }}>P_{c}$, the PM condition for $X_{4}$ cannot be reached, which means that a well-defined attraction of the sidebands towards the birefringence axes is not expected anymore.

From the previous discussion, it is clear that whenever $P_{\text {tot }}<P_{c}$, three distinct sideband polarization attraction processes towards the principal axes of the hibi fiber may occur. Each of these processes drives sidebands with detunings in a specific frequency band that is centered around the corresponding PM frequency; see the summary provided in Table 1 . When acting separately, the processes $X_{(1,2,3,4)}$ give rise to the QLPA effect; i.e., the growing sidebands are attracted towards the hibi fiber axes, as previously discussed. On the other hand, whenever $P_{\text {tot }}>P_{c}$, the competition among the four FWM processes prevents the uniform (i.e., independent of the input pump SOP) attraction of the sidebands towards fixed birefringence axes. In this general case, the full set of equations in Eq. (5) should be solved in order to estimate the output SOP of the sidebands.

\section{POLARIZATION ATTRACTION: ANALYTICS}

The treatment developed in the previous section provides a useful and simple overview of the different FWM processes that lead to the simultaneous amplification and polarization attraction of the sidebands, which may occur whenever $P_{\text {tot }}<P_{c}$. Moreover, the optimal (or PM) sideband detunings corresponding to peak sideband gain were provided. Nevertheless, in order to quantify the gain and corresponding gain bands around the PM frequencies, as well as to provide the actual SOP of the sidebands, the full set of equations in Eq. (5) needs to be solved. As shown in [16], after a proper change of variables, the solution of these equations leads 
Table 1. The Processes $X_{(1,2,3,4)}$ are Listed With Their Corresponding Optimal Detuning Frequency $\Delta \nu_{X i}$ that Leads the Maximum Amplification of the Sidebands, the Attraction of the Sidebands Towards the Birefringence Axes, and the Dependence of the Gain on the Pump Polarization ( $\propto$ Stands for Directly Proportional) ${ }^{a}$

\begin{tabular}{lccc}
\hline Process $X_{i}$ & $\Delta \nu_{X i}$ & Attraction & Gain $\propto$ \\
\hline$X_{(1,2)}$ & $(2 \pi)^{-1}\left(\sqrt{2 \gamma \max (P, Q)\left|\beta_{2}\right|}\right)$ & $i \rightarrow, s \rightarrow(P>Q), i \uparrow, s \uparrow(P<Q)$ & $\max (P, Q)$ \\
$X_{3}$ & $(2 \pi)^{-1}\left(\Delta N\left(c\left|\beta_{2}\right|\right)+\gamma P_{\mathrm{tot}} c \Delta n\right)$ & $i \uparrow, s \rightarrow$ & $\min (P, Q)$ \\
$X_{4}$ & $(2 \pi)^{-1}\left(\Delta n\left(c\left|\beta_{2}\right|\right)-\gamma P_{\mathrm{tot}} c / \Delta n\right)$ & $i \rightarrow, s \uparrow$ & $\min (P, Q)$ \\
\hline
\end{tabular}

${ }^{a}$ It is assumed that $P_{\text {tot }}<P_{c}$. The arrows $\rightarrow$ and $\uparrow$ refer to an attraction towards the $x$ and $y$ axes, respectively.

to an eigenvalue problem-in other words, the evolution of the signal and idler amplitudes along the fiber may be fully described in terms of the eigenvalues and eigenvectors of a matrix $M$; i.e., sidebands, but also to ensure that these sidebands possess a well-defined SOP at the fiber output. In other words, it would be useful if one could operate the FOPA such that the output polarization of the sidebands is (nearly) independent of the

$$
M=\left[\begin{array}{cccc}
-v / 2+K / 2+\gamma P & \gamma P & (2 / 3) \gamma \sqrt{P Q} & (2 / 3) \gamma \sqrt{P Q} \\
-\gamma P & -v / 2-K / 2-\gamma P & -(2 / 3) \gamma \sqrt{P Q} & -(2 / 3) \gamma \sqrt{P Q} \\
(2 / 3) \gamma \sqrt{P Q} & (2 / 3) \gamma \sqrt{P Q} & v 2+K / 2+\gamma Q & \gamma Q \\
-(2 / 3) \gamma \sqrt{P Q} & -(2 / 3) \gamma \sqrt{P Q} & -\gamma Q & v / 2-K / 2-\gamma Q
\end{array}\right]
$$

where $v=2 \pi \Delta \nu \Delta W c$ and $K=4 \pi^{2} \beta_{2} \Delta \nu^{2}$. Let $\lambda=r-i g$ be the eigenvalue of $M$ with the largest positive imaginary part $g$, and $\left[\tilde{A}_{x i}, \tilde{A}_{x s}, \tilde{A}_{y i}, \tilde{A}_{y s}\right]$ the corresponding eigenvector. The associated signal and the idler components are amplified along the fiber according to the expression

$$
\begin{aligned}
A_{j v}(z) & =\left|\tilde{A}_{j v}\right| \exp \left[i \phi_{j v}(z)+i r z\right] \exp (g z)(j=\{x, y\} \\
v & =\{i, s\})
\end{aligned}
$$

where $\phi_{x s}(z)=-\psi_{x s}+\phi_{x p}(z)-(K / 2+v / 2) z, \phi_{x i}(z)=\psi_{x i}+$ $\phi_{x p}(z)-(K / 2-v / 2) z, \quad \phi_{y s}(z)=-\psi_{y s}+\phi_{y p}(z)-(K / 2-v / 2) z$, and $\phi_{y i}(z)=\psi_{y i}+\phi_{y p}(z)-(K / 2+v / 2) z$; here $\psi_{j v}$ is the phase of $\tilde{A}_{j v}$.

As shown by Eqs. (ㅁ) and (7), in general, both pump and sidebands exhibit a $z$-dependent elliptical polarization. In order to characterize the pump polarization, we may define $\quad \alpha_{p}=\arctan \left(\left|A_{x p}(0) \bigvee\right| A_{y p}(0) \mid\right)=\arctan (\sqrt{P / Q}) \quad$ and $\Delta \phi_{p}(z)=\phi_{x p}(z)-\phi_{y p}(z)$. Clearly the elliptical polarization of the pump periodically evolves with distance $z$ among the following limit cases: (i) whenever $\Delta \phi_{p}(z)=2 N \pi$ ( $N$ integer), the pump is linearly polarized at an angle $\alpha_{p}$ with respect to the $y$ axis; (ii) whenever $\Delta \phi_{p}(z)=(2 N+1) \pi$, the pump is linearly polarized at an angle $-\alpha_{p}$ with respect to the $y$ axis; (iii) if $\Delta \phi_{p}(z)=(2 N+1) \pi / 2$, the pump is elliptically polarized with a maximum degree of ellipticity; the axes of the polarization ellipse coincide with the birefringence axes. Similar considerations apply to the idler and signal waves by defining the corresponding angles $\alpha_{i}=\arctan \left(\left|\tilde{A}_{x i} V\right| \tilde{A}_{y i} \mid\right)$ and $\alpha_{s}=$ $\arctan \left(\left|\tilde{A}_{x s} V\right| \tilde{A}_{y s} \mid\right)$, as well as the $z$-dependent phase differences $\Delta \phi_{i}(z)=\phi_{x i}(z)-\phi_{y i}(z)$ and $\Delta \phi_{s}(z)=\phi_{x s}(z)-\phi_{y s}(z)$, respectively.

Whenever the FOPA is used in combination with polarization-sensitive devices (e.g., a polarization dependent receiver), it is not only important to provide gain for the fiber length. Moreover, for practical reasons, it would be desirable to have a signal and an idler that maintain a linear SOP that is aligned with either one of the fiber principal axes of birefringence. On the other hand, as we have seen, in general the output SOP of both signal and idler is elliptical, with a degree of ellipticity that depends on the fiber length. It can be shown that the only situation strictly leading to the parametric amplification of both signal and idler with a linear SOP corresponds to the case of an input pump oriented along either one of the two birefringence axes $\left(\alpha_{p}=0^{\circ}\right.$ or $\left.\alpha_{p}=90^{\circ}\right)$ and propagating in the anomalous dispersion regime. This is a relatively trivial case since both pump and sidebands are polarized along the same axis (limit case of pure scalar propagation).

Nevertheless, as we have underlined in the previous section, whenever the condition Eq. ( 6$)\left(P_{\text {tot }}<P_{c}\right)$ holds, one would expect that if the sideband detuning is nearby the PM frequency of a given process $X_{(1,2,3,4)}$, then the sideband SOP will be strongly attracted towards the birefringence axes of the fiber. If it is so, then the output angles $\alpha_{s}$ and $\alpha_{i}$ for the signal and the idler should remain very close to either $0^{\circ}$ or $90^{\circ}$, virtually independently of the input pump SOP and power, as well as and of the precise value of the sideband frequency detuning within the gain band.

In other words, as we shall see in the next section, whenever $P_{\text {tot }}<P_{c}$, then both signal and idler are strongly attracted towards either the fast or the slow birefringence axis for whatever $\alpha_{p}$ is imposed at the fiber input. As a consequence, the signal and idler SOP remains quasi-linear (i.e., with an ellipticity $\epsilon \ll 1)$ and stable along the distance $z$ so that a QLPA is observed.

In addition, it turns out that the smaller is $P_{\text {tot }}$ with respect to $P_{c}$, the smaller is the range of oscillation of the sideband ellipticity about zero. Clearly the gain coefficient and its bandwidth also depend on $P_{\text {tot }}$ and on $\alpha_{p}$ : the smaller the 
pump power, the lesser the sideband gain $g$. In the next section, we will discuss in detail the cases in which QLPA is observed, and we are going to analyze the corresponding gain characteristics.

\section{NUMERICAL RESULTS}

The most striking manifestation of QLPA in a FOPA based on a hibi fiber is observed in the normal dispersion regime $\left(\beta_{2}>0\right)$. Let us consider a fiber with $\Delta n=3 \cdot 10^{-5}, \gamma=$ $10 \mathrm{~W}^{-1} \mathrm{~km}^{-1}, \beta_{2}=10 \mathrm{ps}^{2} \mathrm{~km}^{-1}$. The pump power $P_{\text {tot }}$ is set to $10 \mathrm{~W}$ in order to respect the condition Eq. (6) $\left(P_{\text {tot }}<P_{c}=\right.$ $25 \mathrm{~W})$, so that the energy-exchange process $X_{4}$ dominates over a gain band that is centered around $\Delta \nu_{X 4}$. In Fig. 1(a), we show the frequency dependence of the parametric gain $g$ for different values of $\alpha_{p}$. Whereas Fig. 2(a) illustrates the corresponding idler angle $\alpha_{i}$ within the gain band, again as a function of $\alpha_{p}$. The signal polarization angle $\alpha_{s}$ is immediately obtained from $\alpha_{i}$ : from a simple analysis of the matrix $M$, it can be shown that, for a given frequency detuning, the relation $\alpha_{s}\left(\alpha_{p}\right)=90^{\circ}-\alpha_{i}\left(90^{\circ}-\alpha_{p}\right)$ holds true. Therefore we only need to plot $\alpha_{i}$ in Fig. 2(a).

The QLPA property clearly emerges in Fig. 2(a); in fact the idler $\alpha_{i}>80^{\circ}\left(\alpha_{s}<10^{\circ}\right)$ throughout the gain band and for any input pump angle $\alpha_{p}$. This means that the idler wave is strongly attracted towards the slow $x$-axis at the fiber output. Correspondingly, the signal is attracted towards the fast $y$-axis. As mentioned in the previous section, the range of oscillation of both $\alpha_{i}$ and $\alpha_{s}$ within the gain band gets progressively smaller as the pump power $P_{\text {tot }}$ is reduced; however, this is obtained at the expense of a corresponding gain reduction. In fact, as predicted in Section 2 (see Table 1), differently from $\alpha_{i}$, the gain $g$ sensitively depends on the pump power $P_{\text {tot }}$
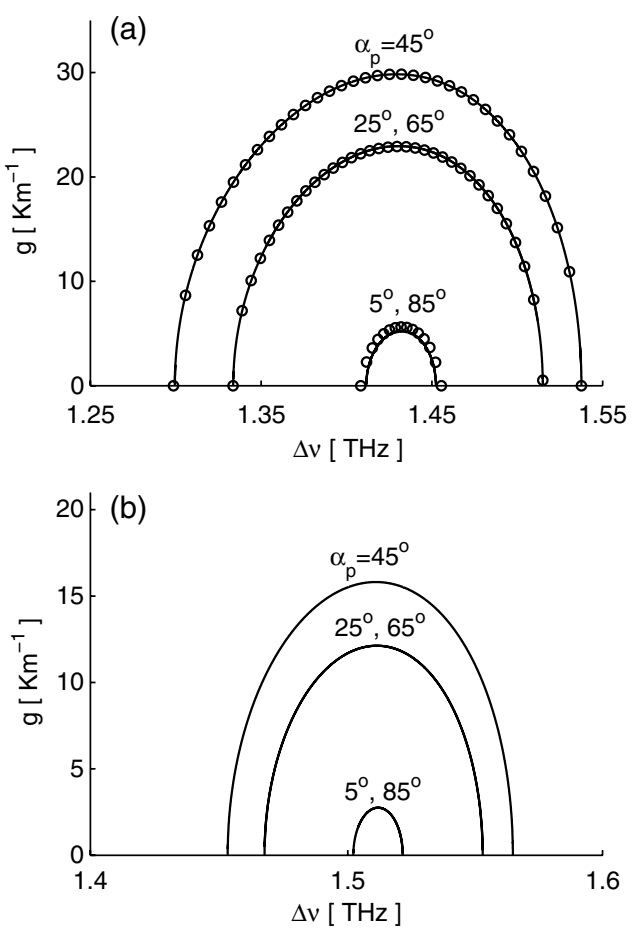

Fig. 1. Gain $g$ in the normal dispersion regime [in (a), $P_{\text {tot }}=10 \mathrm{~W}$; in (b), $P_{\text {tot }}=5 \mathrm{~W}$ ]; the circles in (a) represent the approximated gain of Eq. (A6); $\alpha_{p}=5^{\circ}, 25^{\circ}, 45^{\circ}, 65^{\circ}, 85^{\circ}$.
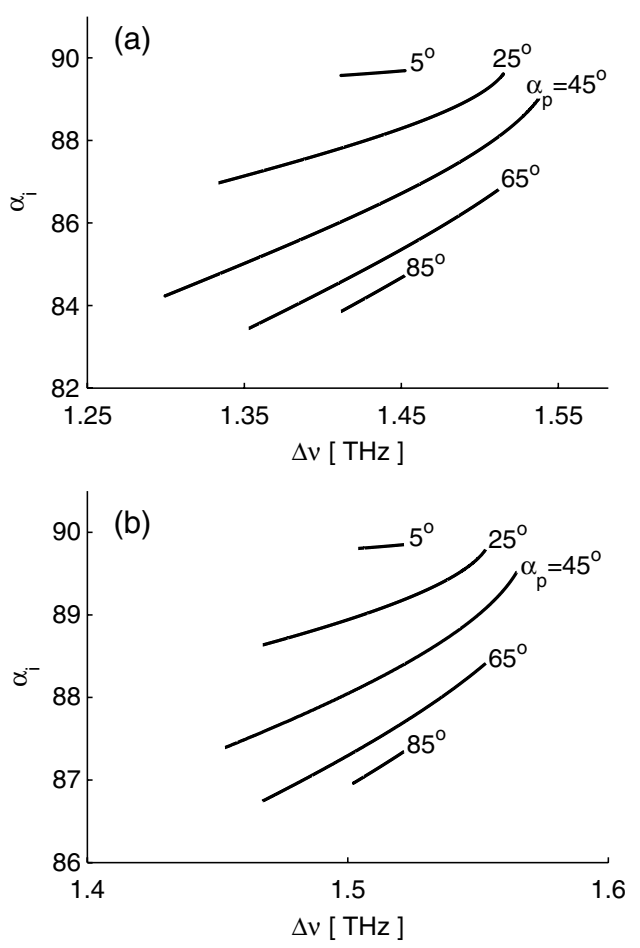

Fig. 2. Angle $\alpha_{i}$ in the normal dispersion regime [in (a), $P_{\text {tot }}=10 \mathrm{~W}$; in (b), $P_{\text {tot }}=5 \mathrm{~W}$ ); $\alpha_{p}=5^{\circ}, 25^{\circ}, 45^{\circ}, 65^{\circ}, 85^{\circ}$.

as well as on the pump SOP or $\alpha_{p}$. This property of the QLPA is clearly shown in Figs. 1(b) and 2(b); here we consider a pump power $P_{\text {tot }}=5 \mathrm{~W}$. As can be seen, on the one hand the QLPA is stronger with respect to the previous case (with $P_{\text {tot }}=$ $10 \mathrm{~W}$ ) since $\alpha_{i}$ remains closer to $90^{\circ}$. On the other hand, the parametric gain is nearly halved for all values of $\alpha_{p}$ with respect the case with $P_{\text {tot }}=10 \mathrm{~W}$.

Although in general the gain $g$, which is the imaginary part of an eigenvalue of $M$, needs to be evaluated by means of a fourth-degree polynomial equation, we found that a very good analytical approximation for $g$ is provided by the expressions for $g_{\text {app }}$ and $g_{\text {app2 }}$, which are provided in Appendix A. These formulas lead to simple analytical expressions for the lower and upper cut-off frequencies of the gain band, as well as for the peak gain $g_{\max }$. In particular, the expression for $g_{\text {app }}$ is derived in Appendix A by means of a perturbation expansion of the matrix $M$, which permits us to analytically show that the signal and the idler are strongly attracted towards the $x$-and $y$-axes, respectively. That is, Appendix A provides a full mathematical support to the conclusions of Section $\underline{2}$, which were based on simple phase-matching arguments. The expression for $g_{\text {app }}$ estimates the peak gain at the PM frequencies simply as $(2 / 3) \gamma \sqrt{P Q}$ [in good accordance with the results in Fig. 1(a)]. As shown by the empty circles in Fig. 1(a), $g_{\text {app2 }}$ also provides an excellent simple analytical estimation of the parametric gain as a function of the various physical parameters. It can be shown that, whenever condition (6) is satisfied, a lower cut-off frequency always exists for the gain band in normal dispersion. Moreover, as can be seen in Fig. 1 and as it was predicted in Section $\underline{2}$, the sideband detuning $\Delta \nu_{\max }$ remains virtually independent of $\alpha_{p}$ [it is equal to $1.43 \mathrm{THz}$ in Fig. 1(a), which is in perfect agreement with the $\Delta \nu_{X 4}$ value in Table 1 ], and it represents the PM frequency 
detuning for the $X_{4}$ parametric amplification process exhibiting the QLPA property.

In the anomalous dispersion regime $\left(\beta_{2}<0\right)$, the vector FWM process is more complex than in the case of normal dispersion, yet it is still possible to obtain the QLPA property for certain frequency ranges of the sideband detuning. For anomalous dispersion, there may be two distinct eigenvalues of $M$, say $\lambda_{1}=r_{1}-i g_{1}$ and $\lambda_{2}=r_{2}-i g_{2}$ with a positive imaginary component. Correspondingly, two frequency bands, say $B_{1}$ and $B_{2}$, exist where either $g_{1}>0$ or $g_{2}>0$. Since $B_{2} \subseteq B_{1}$ and $g_{1} \geq g_{2}$, let us consider the sideband amplification process associated with the gain $g_{1}=g$ and the corresponding bandwidth $B_{1}$. In our examples, we used the same fiber parameters as in the previously discussed case of the normal dispersion regime, but changed the sign of the group velocity dispersion coefficient (i.e., we set $\beta_{2}=-10 \mathrm{ps}^{2} \mathrm{~km}^{-1}$ ).

In Figs. $\underline{3}$ and $\underline{4}$, we show the gain $g$ and the idler angle $\alpha_{i}$ of the corresponding eigenvector. By analyzing the matrix $M$, it can be seen that, when the condition (6) is satisfied, for any pump angle $\alpha_{p}$ a band gap exists within the gain band $B_{1}$. Namely, we may distinguish between a low-frequency band $B_{1, L}$ with no lower cut-off frequency, and a high-frequency band $B_{1, H}$. See, for example, Fig. 3: for $\alpha_{p}=40^{\circ}, B_{1, L}$ extends from zero frequency detuning up to $\Delta \nu<0.76 \mathrm{THz}$; whereas $B_{1, H}$ spans the frequency range $1.64 \mathrm{THz}<\Delta \nu<1.83 \mathrm{THz}$. The corresponding band gap is $0.76 \mathrm{THz}<\Delta \nu<1.64 \mathrm{THz}$. Note that the frequency bands $B_{1, L}, B_{1, H}$, and $B_{2}$ are associated with the different FWM processes that were described in Section 2. Namely, $B_{1, H}$ is associated with the process $X_{3}$, whereas $B_{1, L}$ and $B_{2}$ are associated with $X_{1}$ and $X_{2}$, which may coexist over a certain frequency band. Therefore the gain $g_{1}=g$ in $B_{1, L}$ originates from the process $X_{1}\left(X_{2}\right)$ when $P>Q$ $(P<Q)$. As we can see from Fig. 4 , for sideband detunings within $B_{1, L}$ and for any pump angle $\alpha_{p}<45^{\circ}$, both idler and signal eigenvector polarizations are strongly attracted towards the slow $x$-axis. On the other hand, whenever $\alpha_{p}>$ $45^{\circ}$, signal and idler are attracted towards the fast $y$-axis. Therefore, as it was predicted in Section 2 , for sideband detunings within $B_{1, L}$ the QLPA is no longer pump independent, since the fiber axis of attraction depends on the range of variation of $\alpha_{p}$, i.e., on which process between $X_{1}$ and $X_{2}$ provides the largest gain.

Note that in the limit case where $\alpha_{p} \approx 45^{\circ}, g_{2} \approx g_{1}$ and $B_{2} \approx B_{1, L}$ : in fact, $P \approx Q$ so that neither of the two processes

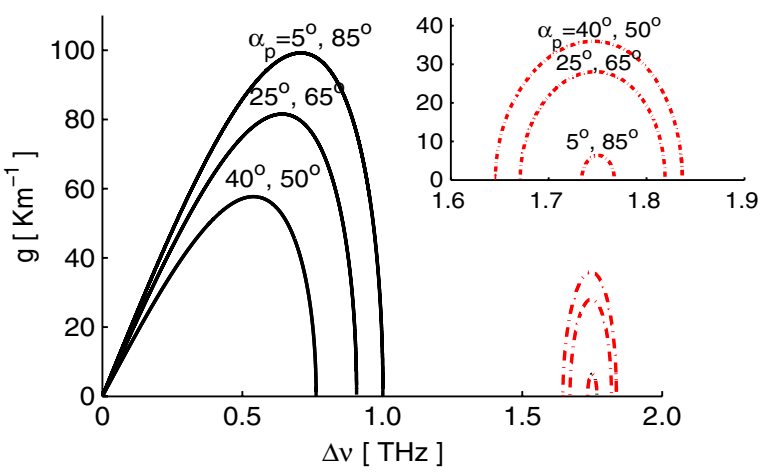

Fig. 3. (Color online) Sideband gain $g$ in the anomalous dispersion regime $\left(P_{\text {tot }}=10 \mathrm{~W}\right)$. Black solid line for band $B_{1, L}$, red dash-dot line for $B_{1, H} ; \alpha_{p}=5^{\circ}, 25^{\circ}, 45^{\circ}, 50^{\circ} 65^{\circ}, 85^{\circ}$. The inset provides a zoom for the band $B_{1, H}$ in the frequency range $1.6 \mathrm{THz} \leq \Delta \nu \leq 1.9 \mathrm{THz}$.

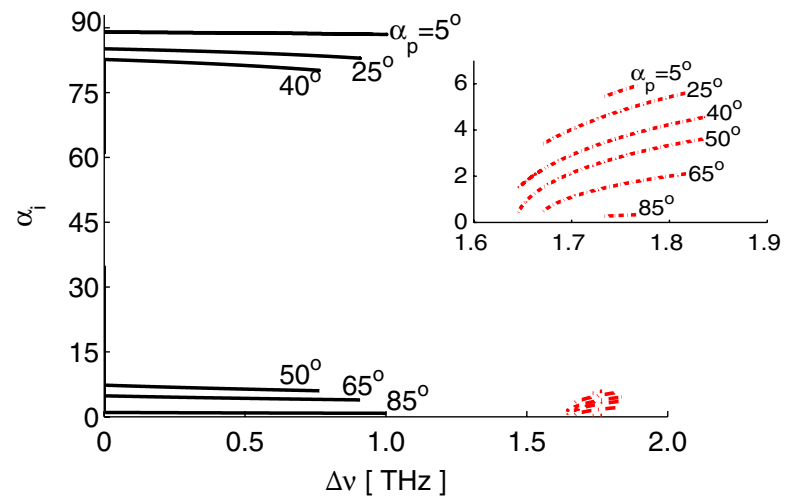

Fig. 4. (Color online) Angle $\alpha_{i}$ in the anomalous dispersion regime $\left(P_{\text {tot }}=10 \mathrm{~W}\right)$. Black solid line for band $B_{1, L}$, red dash-dot line for $B_{1, H}$; $\alpha_{p}=5^{\circ}, 25^{\circ}, 40^{\circ}, 50^{\circ} 65^{\circ}, 85^{\circ}$. The inset provides a zoom for the band $B_{1, H}$ in the frequency range $1.6 \mathrm{THz} \leq \Delta \nu \leq 1.9 \mathrm{THz}$.

$X_{1}$ and $X_{2}$ are clearly dominant over the other. In this situation, the sideband polarization angles $\alpha_{i}$ and $\alpha_{s}$ no longer reach a steady-state value, but they oscillate along the fiber, and their specific value at any distance $z$ depends on the input sideband (or seeding) conditions at $z=0$.

On the other hand, for sideband detunings within $B_{1, H}$, there is no overlap with $B_{2}$; hence a single amplified eigenvector exists in this case. As can be observed in Fig. 4 and as was anticipated in Section 2, for a frequency detuning within $B_{1, H}$, the idler experiences a proper QLPA towards the fast axis, whereas the signal experiences QLPA towards the slow axis. Namely, polarization attraction occurs nearly independently of the pump angle, and even for $\alpha_{p} \approx 45^{\circ}$. As it occurs in the normal dispersion case, the analytical formula $g_{\text {app }}$ for the parametric gain described in Appendix A applies in $B_{1, H}$. Note that the derivation of this formula in Appendix A also provides the formal demonstration that signal and idler are strongly attracted towards the $x$-and the $y$-axes, respectively. As anticipated in Section 2 and shown by Fig. 4 , the optimum (peak gain) detuning frequency $\Delta \nu_{\max }=\Delta \nu_{X 3}$ is virtually independent of $\alpha_{p}: \Delta \nu_{\max }=1.75 \mathrm{THz}$ in Fig. 4 , which is in perfect accordance with $\Delta \nu_{X 3}$ reported in Table 1 . Note that $g_{\text {app }}$ does not provide a good estimation of the parametric gain in $B_{1, L}$ : in this frequency band, the full fourth-degree polynomial equation should be used. Yet we may still exactly calculate the corresponding $\Delta \nu_{\max }=\max \left(\Delta \nu_{X 1}, \Delta \nu_{X 2}\right)$ : as expected, the parametric gain is proportional to $\max (P, Q)$, as can be seen in Fig. 4. For a better appreciation of the peculiarity of the QLPA property, in Figs. 5 and 6 we display an example where Eq. (6) is not respected; here the fiber parameters are the same as in the normal dispersion case of Figs. 1(a) and 2(a),

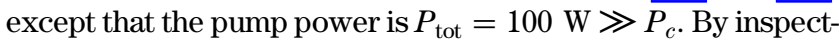
ing Figs. 5 and 6 , it is clear that the QLPA property is no longer present all over the parametric gain band. This is due to the lack of a prevailing phase-matched energy-exchange process, as explained in Section 2. As a matter of fact, $\alpha_{i}$ sweeps from nearly $25^{\circ}$ to $85^{\circ}$ depending on $\Delta \nu$ and $\alpha_{p}$ : the sideband polarization has a significant ellipticity, and it periodically evolves upon propagation, as outlined in the previous section.

In order to provide a clear display of the QLPA property, we conclude this section by presenting three specific examples where the original CNLSEs governing the FWM (or vector MI) process are solved [see Eq. (1)]. In this way, we illustrate how the QLPA is reached at the fiber output, 


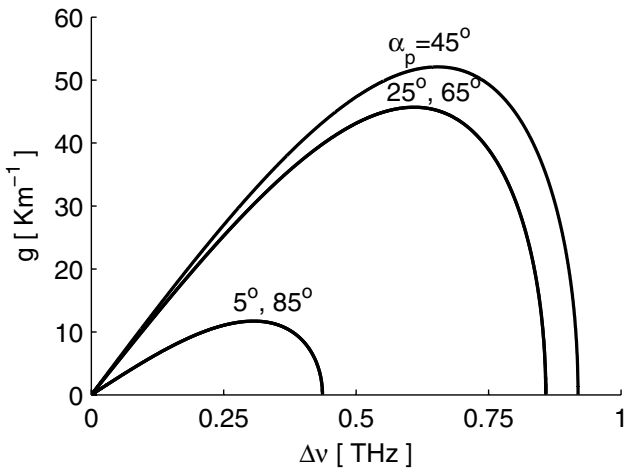

Fig. 5. Sideband gain $g$ in the normal dispersion regime $\left(P_{\text {tot }}=100 \mathrm{~W}\right) ; \alpha_{p}=5^{\circ}, 25^{\circ}, 45^{\circ}, 65^{\circ}, 85^{\circ}$.

nearly independently of the input polarizations of both pump and sidebands. Let us consider again the same fiber parameters and input pump power that we used for the first example of the normal dispersion case in Figs. 1(a)-2(a): we fix here the input sidebands power at $1.0 \mu \overline{\mathrm{W}}$, and their frequency detuning at $\Delta \nu=1.43 \mathrm{THz}$, which guarantees peak gain for any $\alpha_{p}$ [see Fig. 1(a)]. We are going to represent the output SOP of the waves in the Stokes space by means of the unit Stokes vectors $\vec{S}_{j}=\left[S_{1 j}(z)=S_{0 j}^{-1}\left(A_{x j}^{*}(z) A_{y j}(z)+A_{x j}(z)\right.\right.$ $\left.A_{y j}^{*}(z)\right), S_{2 j}(z)=S_{0 j}^{-1}\left(i A_{x j}^{*}(z) A_{y j}(z)-i A_{x j}(z) A_{y j}^{*}(z)\right), S_{3 j}(z)=$ $\left.S_{0 j}^{-1}\left(\left|A_{x j}(z)\right|^{2}-\left|A_{y j}(z)\right|^{2}\right)\right](j=i, p, s)$, being $S_{0 j}=\left(\left|A_{x j}(z)\right|^{2}+\right.$ $\left.\left|A_{y j}(z)\right|^{2}\right)^{1 / 2}$.

In a first example, we fix the input pump Stokes vector $\vec{S}_{p}(0)=[1,0,0]$; i.e., we set the linear pump polarization along the direction $\alpha_{p}=45^{\circ}$. Moreover, we solve the CNLSEs for a set of 225 different input sidebands Stokes vectors $\vec{S}_{i, n}(0)=$ $\vec{S}_{s, n}(0)(1 \leq n \leq 225)$, which are uniformly distributed over the Poincaré sphere [see Fig. 7(a)], in order to span the sideband input polarization across all possible values. We set the fiber length at $L=100 \mathrm{~m}$. In Fig. 7(b), we show the resulting output SOP of the idler wave: the figure clearly shows that all of the 225 output Stokes vectors $\vec{S}_{i, n}(L)$ are strongly attracted to the vector $[0,0,1]$, which corresponds to a linear SOP oriented along the $x$-axis. This means that a quasi-linear polarization along the $x$-axis, nearly independent of the input polarization of the sidebands, is reached at the fiber output. The degree of polarization $\mathrm{DOP}_{v}(z)$ that measures the strength of the attraction is calculated as $\operatorname{DOP}_{v}(z)=<S_{1 v}(z)>^{2}+<S_{2 v}(z)>^{2}+$ $<S_{3 v}(z)>^{2}(v=\{i, s, p\})$, where the average values $<S_{k v}(z)>$,

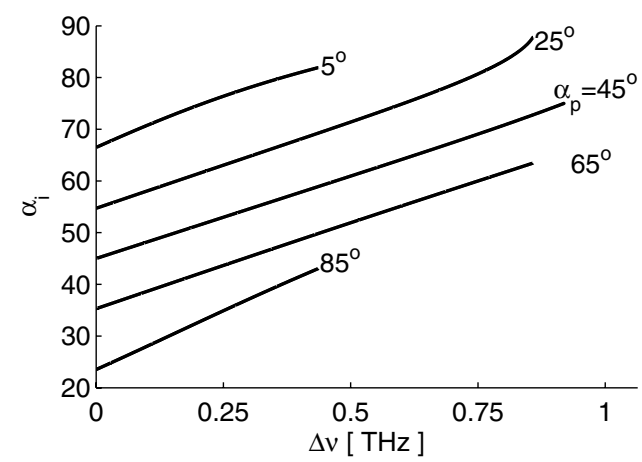

Fig. 6. Angle $\alpha_{i}$ in the normal dispersion case discussed in Section 3 $\left(P_{\text {tot }}=100 \mathrm{~W}\right) ; \alpha_{p}=5^{\circ}, 25^{\circ}, 45^{\circ}, 65^{\circ}, 85^{\circ}$. The pump-independent QLPA is not reached as $\alpha_{i}$ sweeps from nearly $25^{\circ}$ to $85^{\circ}$ depending on the detuning frequency $\Delta \nu$ and on the input pump angle $\alpha_{p}$.
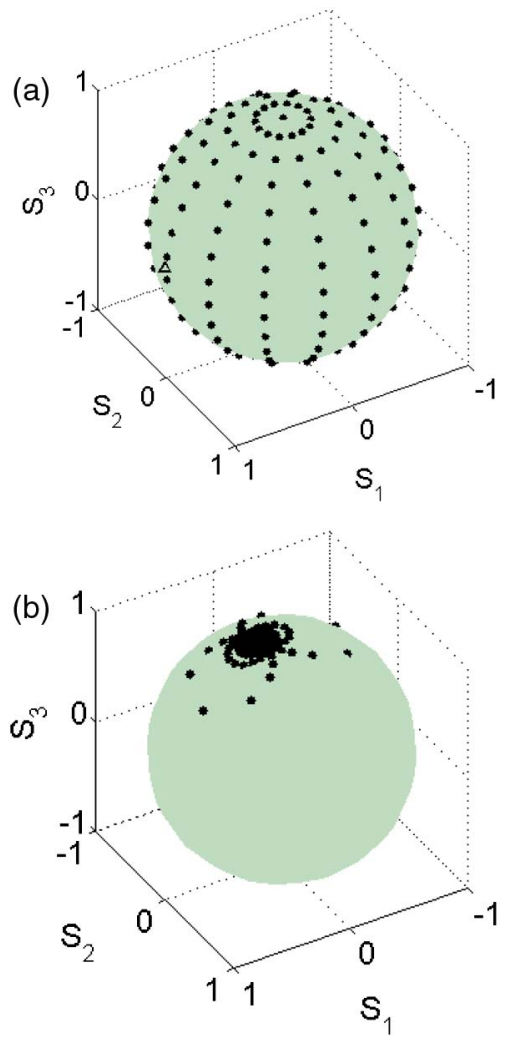

Fig. 7. (Color online) (a) Fixed input unit Stokes vector of the pump $\vec{S}_{p}(0)=\left[S_{1}=1, S_{2}=0, S_{3}=0\right]$ (empty triangle) and 225 input unit Stokes vectors of the sidebands $\vec{S}_{i, n}(0)=\vec{S}_{s, n}(0)$ (filled circles) are shown on the Poincaré sphere $(1 \leq n \leq 225)$; (b) output unit Stokes vectors of the idler $\vec{S}_{i, n}(L)$ (filled circles) after fiber propagation (fiber length $L=100 \mathrm{~m}$ )

$k=(1,2,3)$ are calculated such as in [18], and is as high as $\operatorname{DOP}_{i}(L)=0.98$ at the fiber output; this shows that the attraction is very strong after just $100 \mathrm{~m}$ of hibi nonlinear fiber.

Let us now consider a second example in which the parameters are the same as before, except that the input idler is set to zero, as it occurs in typical FWM experiments. In Fig. 8, the resulting output SOP of the idler is shown: it is possible to observe that the attraction along the $x$-axis is even stronger than the previous case. This can be explained in terms of a larger projection of the initial sideband amplitudes on the

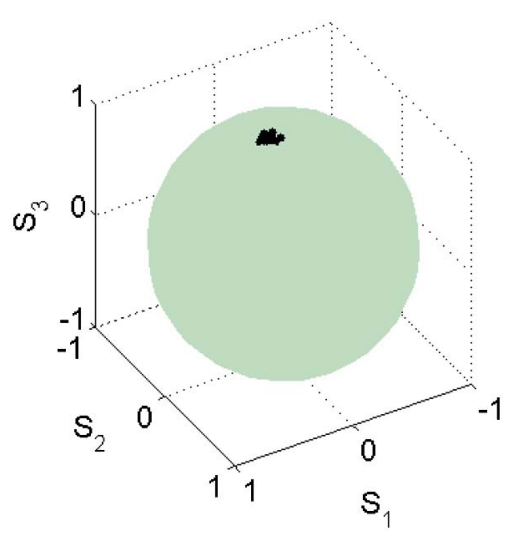

Fig. 8. (Color online) Output unit Stokes vectors of the idler $\vec{S}_{i, n}(L)$ (filled circles) after fiber propagation (fiber length $L=100 \mathrm{~m}$ ). The input idler is null; the input unit Stokes vector of the pump and of the signal are the same as in Fig. 7 (a). 

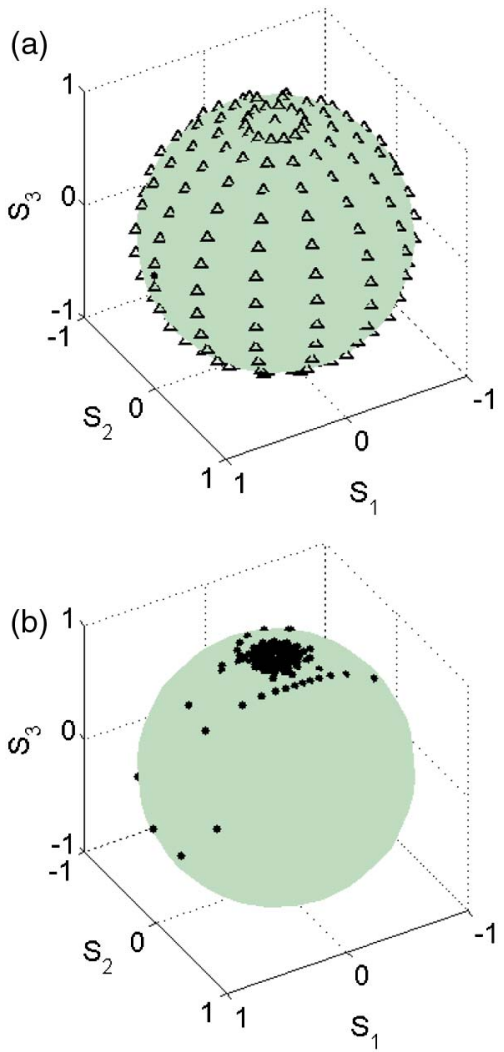

Fig. 9. (Color online) (a) Fixed input unit Stokes vectors of the sidebands $\vec{S}_{i, n}(0)=\vec{S}_{s, n}(0)=[1,0,0]$ (filled circle) and 225 input unit Stokes vectors of the pump (empty triangles) are shown on the Poincaré sphere; (b) output unit Stokes vectors of the idler $\vec{S}_{i, n}(L)$ (filled circles) after fiber propagation (fiber length $L=300 \mathrm{~m}$ ).

eigenvector Eq. (7) that is associated with exponential growth with distance.

To conclude, we consider a third example where both the input Stokes vectors of the idler and signal $\vec{S}_{i, n}(0)=\vec{S}_{s, n}(0)=$ $[1,0,0]$ are kept equal and fixed, and we span our CNLSE simulations over a set of 225 input pump Stokes vectors $\vec{S}_{p, n}(0)$ $(1 \leq n \leq 225)$, uniformly distributed over the Poincaré sphere, so that all possible input pump polarization states are varied [see Fig. 9(a)]. As previously discussed, the parametric or Michigan gain depends on the input pump polarization (see Fig. 1). Therefore in this case we tripled the fiber length $(L=300 \mathrm{~m})$ in order to guarantee a sufficient gain for most sidebands (hence their corresponding attraction towards the birefringence axes) as the input pump Stokes vector is varied. The results of Fig. 9 clearly show once again that most of the 225 output Stokes vectors of the idlers $\vec{S}_{i, n}(L)$ experience significant attraction towards the vector $[0,0,1]$. Figure 9 also reveals that some of the output idler vectors are not well attracted towards the $x$-axis of the fiber, since the corresponding MI gain is not strong enough to permit an effective attraction in just $300 \mathrm{~m}$ of fiber. In any case, by averaging over all input pump polarizations, we obtain that the $\operatorname{DOP}_{i}(L)=0.97$, which indicates that the average attraction is still very strong in spite of the variation of the input pump SOP.

\section{CONCLUSIONS}

In this work, we have analyzed the polarization attraction properties of a signal and an idler that are parametrically amplified by a copropagating pump in a hibi fiber. In general, the sidebands exhibit an elliptical and spatially varying SOP that also depends on the pump SOP and on their frequency detuning from the pump. Nevertheless we pointed out that some specific conditions exist that permit the generation of sidebands with a stable SOP that remains oriented along one of the principal axes of the fiber, virtually irrespective of (i) the input sideband SOP; (ii) the input pump SOP; (iii) the input sideband frequency detuning from the pump (provided the detuning falls within the gain band); (iv) the input pump power, as long as Eq. (ㅁ) is satisfied. We named such an interesting property as a pump-independent QLPA, or pumpindependent QLPA. In Section 2 we observed that QLPA is related to some peculiar energy-exchange processes between pump and sideband photons that turn to be dominant in a particular gain band. Indeed we have seen that, whenever the pump propagates in the normal dispersion regime and Eq. (6) holds, QLPA of the idler (signal) occuring along the slow (fast) birefringence axis is obtained. In this case, the sideband detuning band has lower and upper cut-off frequencies, and we provided a simple analytical formula for the parametric gain. With a pump in the anomalous dispersion regime and if condition Eq. (6) holds, the sideband gain exhibits a band gap that separates low and high-frequency sub-bands with very different polarization attraction properties. In the low-frequency sub-band, both sidebands experience a QLPA towards either the fast or the slow axis, whenever the input pump angle $\alpha_{p}$ is greater or smaller than $45^{\circ}$, respectively. On the other hand, proper QLPA is observed in the high-frequency sub-band, where the idler (signal) is always strongly attracted towards the fast (slow) axis.

Polarization attraction and control for the generated signal and idler in FOPAs has interesting potential applications when used for frequency-conversion and phase-sensitive amplification in combination with polarization-sensitive optical processing devices (e.g., a heterodyne receiver). Moreover, since a codirectional pump is used, FOPA repolarizers based on hibi fibers may potentially lead to compensating ultrafast input polarization fluctuations, the upper limit to its speed being set by the relatively large differential group delay between the birefringence axes or PMD. Although parametric polarizers such as the one described here suffer from output RIN, we anticipate that substantial RIN suppression could be obtained when saturating the gain by operating the amplifier in the depleted pump regime, as it has been observed with Raman polarizers [19]. Another possibility of substantially reducing the output RIN could be provided by performing a bit-synchronous polarization scrambling of the input signal (or idler), so that the parametric gain is averaged over the pulse profile. Such a scheme has proven to be effective in the case of Raman polarizers [20]. From the practical point of view, it would also be very interesting to consider the extension of our study to the case of FWM-induced polarization attraction in optical fibers with randomly varying birefringence. This situation requires the derivation of the corresponding averaged equations as in [9], and will be the subject of a subsequent report.

\section{APPENDIX A}

In this appendix we derive, by means of a perturbation approach that is valid whenever the condition Eq. (6) is verified, a simple analytical expression for the FWM (or $\bar{M}$ ) sideband 
gain in a frequency detuning band around $\Delta \nu_{X 3}$ or $\Delta \nu_{X 4}$, respectively. We also show that under the same conditions, the growing sidebands are strongly attracted towards opposite linear birefringence axes according to the configurations described in Section 2 and summarized in Table 1 . This derivation thus provides a formal a mathematical demonstration of the pump-independent QLPA effect, which usefully completes the physical arguments presented in Section 2 , basing ourselves on the PM conditions of the four-photon energyexchange processes $X_{3}$ and $X_{4}$.

Let us start from the pump-independent QLPA effect based on $X_{4}$, whose PM condition reads as

$$
\beta_{2}\left(2 \pi \Delta \nu_{X 4}\right)^{2}-(\Delta W c)\left(2 \pi \Delta \nu_{X 4}\right)+\gamma P_{\text {tot }}=0 .
$$

Under the condition $P_{\text {tot }}<P_{c}$, the term $\gamma P_{\text {tot }}$ represents a relatively small perturbation with respect to the other terms in Eq. (1ㅡ). Thus $\gamma P_{\text {tot }} \ll v\left(\Delta \nu_{X 4}\right)$ and $\gamma P_{\text {tot }} \ll K\left(\Delta \nu_{X 4}\right)$; in general, $\gamma P_{\text {tot }} \ll v(\Delta \nu)$ and $\gamma P_{\text {tot }} \ll K(\Delta \nu)$ even for $\Delta \nu=$ $\Delta \nu_{X 4}+\partial \nu$. Since $(P-Q) \leq P_{\text {tot }}$, we may conclude that the terms Eq. (A3) and Eq. (A4) can be considered as small perturbations with respect to the terms $-v(\Delta \nu) / 2-K(\Delta \nu) / 2$ and $v(\Delta \nu) / 2+K(\Delta \nu) / 2$ in the second and the third component of the main diagonal of matrix $M$. The same holds true for the terms $P, Q$, and $(2 / 3) \sqrt{P Q}$, as they all remain smaller than $P_{\text {tot }}$. We may thus rewrite $M$ as the sum of the following matrices $\tilde{M}$ and $\partial M$, where $\partial M$ represents a perturbation matrix with respect to $\tilde{M}$ :

$$
\begin{aligned}
\tilde{M} & =\left[\begin{array}{cccc}
0 & 0 & 0 & 0 \\
0 & -\mho / 2-K / 2 & 0 & 0 \\
0 & 0 & v 2+K / 2 & 0 \\
0 & 0 & 0 & 0
\end{array}\right], \\
\partial M & =\left[\begin{array}{cccc}
M(1,1) & \gamma P & (2 / 3) \gamma \sqrt{P Q} & (2 / 3) \gamma \sqrt{P Q} \\
-\gamma P & -\gamma P & -(2 / 3) \gamma \sqrt{P Q} & -(2 / 3) \gamma \sqrt{P Q} \\
(2 / 3) \gamma \sqrt{P Q} & (2 / 3) \gamma \sqrt{P Q} & \gamma Q & \gamma Q \\
-(2 / 3) \gamma \sqrt{P Q} & -(2 / 3) \gamma \sqrt{P Q} & -\gamma Q & M(4,4)
\end{array}\right] .
\end{aligned}
$$

From the definition of $v(\Delta \nu)=2 \pi \Delta \nu \Delta N c$ and $K(\Delta \nu)=$ $4 \pi^{2} \beta_{2} \Delta \nu^{2}$ given in Section 3, the PM condition at the frequency $\Delta \nu_{x 4}$ (whose value is reported in Table 1) can be rewritten as $-v\left(\Delta \nu_{X 4}\right)+K\left(\Delta \nu_{X 4}\right)+\gamma P_{\text {tot }}=0$ or, equivalently, $-v\left(\Delta \nu_{X 4}\right) / 2+K\left(\Delta \nu_{X 4}\right) / 2+\gamma P / 2+\gamma Q / 2=0$. Let us now consider a small perturbation $\partial \nu$ to the sideband PM frequency; i.e., we set $\Delta \nu=\Delta \nu_{X 4}+\partial \nu$. Then by means of a Taylor series development truncated at the first order, we obtain

$$
\begin{aligned}
&-v(\Delta \nu) / 2+K(\Delta \nu) / 2+\gamma P / 2+\gamma Q / 2 \\
&= \overbrace{-v\left(\Delta \nu_{X 4}\right) / 2+K\left(\Delta \nu_{X 4}\right) / 2+P / 2+Q / 2}^{=0} \\
&--\pi \partial \nu \Delta n c+4 \pi^{2} \partial \nu \Delta \nu_{X 4} \beta_{2} \\
&=-\pi \partial \nu \Delta n c+4 \pi^{2} \partial \nu \Delta \nu_{X 4} \beta_{2} .
\end{aligned}
$$

By using Eq. (A2), the first and the fourth term on the main diagonal of the matrix $M$ can be rewritten as

$$
\begin{aligned}
-v(\Delta \nu) / 2+K(\Delta \nu) / 2+\gamma P= & -\pi \partial \nu \Delta w c+4 \pi^{2} \partial \nu \Delta \nu_{X 4} \beta_{2} \\
& +\gamma(P-Q) 2,
\end{aligned}
$$

$$
\begin{aligned}
v(\Delta \nu) 2-K(\Delta \nu) \mathcal{2}-\gamma Q= & +\pi \partial \nu \Delta W c-4 \pi^{2} \partial \nu \Delta \nu_{X 4} \beta_{2} \\
& +\gamma(P-Q) 2 .
\end{aligned}
$$

Here $M(1,1)$ and $M(4,4)$ are provided by the right-hand sides of Eqs. (A2) and (A3), respectively. The matrix $\tilde{M}$ exhibits a double degenerate zero eigenvalue. The two corresponding independent eigenvectors are $v_{01}=[1,0,0,0]^{T}$ and $v_{02}=$ $[0,0,0,1]^{T}$. By applying the perturbation theory for degenerate states, the first-order correction $\lambda$ to the zero eigenvalue turns out to be the solution of $\operatorname{Det}(D)=0$ (Det stands for determinant), where $D$ is the following matrix:

$$
\left[\begin{array}{cc}
\lambda-<v_{01}, \partial M v_{01}> & -<v_{01}, \partial M v_{02}> \\
-<v_{02}, \partial M v_{01}> & \lambda-<v_{02}, \partial M v_{02}>
\end{array}\right]
$$

Here the brackets $<>$ stand for scalar product. The estimated gain $g_{\text {app }}$ is the imaginary part of $\lambda$; that is

$$
g_{\text {app }}=\left[(4 / 9) \gamma^{2} P Q-\left(\Delta \nu-\Delta \nu_{X 4}\right)^{2}\left(4 \pi^{2} \beta_{2} \Delta \nu_{X 4}-\pi \Delta n c\right)^{2}\right]^{1 / 2} .
$$

Note that $g_{\text {app }}$ as function of $\Delta \nu$ in Eq. (A5) is a semiellipse centered at $\Delta \nu_{X 4}$, and it reaches its peak value $g_{\text {app,max }}=$ $(2 / 3) \gamma \sqrt{P Q}$ in correspondence to the PM frequency $\Delta \nu_{X 4}$. The so called "zeroth" order correction $v_{0}$ to the eigenvector associated to $\lambda$ is a linear combination of $v_{01}$ and $v_{02}$, and it reads as $\left[c_{1}, 0,0, c_{2}\right]^{T}$, where $c_{1}$ and $c_{2}$ are the respective projection coefficients. The vector $v_{0}$ turns out to provide a good approximation of $\left[\tilde{A}_{x i}, \tilde{A}_{x s}, \tilde{A}_{y i}, \tilde{A}_{y s}\right]^{T}$ (see Section 3 ) whenever Eq. (A5) is satisfied. This demonstrates that under a first-order approximation, all of the sideband energy is maintained along 
the idler $x$-component and the signal $y$-component, which confirms the pump-independent QLPA property of the sidebands numerically observed in Section 4 in association with the $X_{4}$ process [under the condition Eq. (6)]. A similar perturbation analysis can be carried out for the pump-independent QLPA associated with the $X_{3}$ process in the anomalous dispersion regime. Indeed, the formula Eq. (A5) for $g_{\text {app }}$ still applies with the substitution of $\Delta \nu_{X 4}$ with $\Delta \nu_{X 3}$ and of $\Delta n$ with $-\Delta n$. In this case the "zeroth" order correction to the eigenvector reads as $\left[0, c_{1}, c_{2}, 0\right]^{T}$, which means that most of the sideband energy is carried by the signal $x$-component and the idler $y$-component. Again, the perturbation analysis confirms the pump-independent QLPA property of the sidebands that was observed in the previous sections and associated with the process $X_{3}$ [under the condition Eq. (6)]. An even better (but more complex) analytical formula for the sideband gain, which is valid whenever the condition Eq. (ㅁ) is satisfied, may be obtained by directly calculating the fourth-degree polynomial equation for the eigenvalues of the matrix $M$. By neglecting the two main orders, a second-order polynomial equation is obtained, which can be easily solved. This procedure turns out to provide the following very good approximation $g_{\mathrm{app} 2}$ for the sideband gain:

$$
\begin{aligned}
g_{\mathrm{app} 2} & =\sqrt{4 a c-b^{2},} \\
a & =-2\left(\gamma P_{\mathrm{tot}} K+K^{2}+v^{2}\right), \\
b & =4 v K \gamma(P-Q), \\
c & =2 \gamma P_{\mathrm{tot}}\left(K^{3}-K v^{2}\right)+\left(v^{2}-K^{2}\right)^{2}-(209) \gamma^{2} P Q K^{2} .
\end{aligned}
$$

\section{ACKNOWLEDGMENTS}

We are grateful to V. V. Kozlov for his many helpful suggestions and his critical reading of the manuscript, and we thank A. Bononi for a stimulating discussion. This work was carried out with support from the PRIN2008 (Programma di Ricerca Scientifica di Rilevante Interesse Nazionale) project "Nonlinear cross-polarization interactions in photonic devices and systems."

\section{REFERENCES}

1. M. Reimer, D. Dumas, G. Soliman, D. Yevick, and M. OSullivan, "Polarization evolution in dispersion compensation modules," presented at the Optical Fiber Communications/National Fiber Optic Engineers Conference (OFC/NFOEC), San Diego, CA, USA, March 2009, paper OWD4.

2. R. Noe, H. Heidrich, and D. Hoffmann, "Endless polarization control systems for coherent optics," J. Lightwave Technol. 6, 1199-1208 (1988).
3. B. Koch, R. Noe, V. Mirvoda, H. Griesser, S. Bayer, and H. Wernz, "Record $59 \mathrm{krad} / \mathrm{s}$ polarization tracking in $112 \mathrm{~Gb} / \mathrm{s} 640-\mathrm{km}$ PDM-RZ-DQPSK transmission," IEEE Photon. Technol. Lett. 22, 1407-1409 (2010).

4. A. Zadok, E. Zilka, A. Eyal, L. Thevenaz, and M. Tur, "Vector analysis of stimulated Brillouin scattering amplification in standard single-mode fibers," Opt. Express 16, 21692-21707 (2008).

5. M. Martinelli, M. Cirigliano, M. Ferrario, L. Marazzi, and P. Martelli, "Evidence of Raman-induced polarization pulling," Opt. Express 17, 947-955 (2009).

6. V. V. Kozlov, Javier Nuño, J. D. Ania-Castañón, and S. Wabnitz, "Theory of fiber optic Raman polarizers," Opt. Lett. 35, 3970-3972 (2010).

7. V. V. Kozlov, K. Turitsyn, and S. Wabnitz, "Nonlinear repolarization in optical fibers: polarization attraction with copropagating beams," Opt. Lett. 36, 4050-4052 (2011).

8. S. Pitois, A. Picozzi, G. Millot, H. R. Jauslin, and M. Haelterman, "Polarization and modal attractors in conservative counterpropagating four-wave interaction," Europhys. Lett. 70, 88-94 (2005).

9. V. V. Kozlov, J. Nun̄o, and S. Wabnitz, "Theory of lossless polarization attraction in telecommunication fibers," J. Opt. Soc. Am. B 28, 100-108 (2011).

10. S. Pitois, G. Millot, and S. Wabnitz, "Nonlinear polarization dynamics of counterpropagating waves in an isotropic optical fiber: theory and experiments," J. Opt. Soc. Am. B 18, 432-443 (2001)

11. S. Pitois, J. Fatome, and G. Millot, "Polarization attraction using counter-propagating waves in optical fiber at telecommunication wavelengths," Opt. Express 16, 6646-6651 (2008).

12. J. Fatome, S. Pitois, P. Morin, and G. Millot, "Observation of light-by-light polarization control and stabilization in optical fibre for telecommunication applications," Opt. Express 18 15311-15317 (2010).

13. S. Wabnitz, "Modulational polarization instability of light in a nonlinear birefringent dispersive medium," Phys. Rev. 38, 2018-2021 (1988).

14. J. E. Rothenberg, "Modulational instability for normal dispersion," Phys. Rev. 42, 682-685 (1990).

15. P. D. Drummond, T. A. B. Kennedy, J. M. Dudley, R. Leonhardt, and J. D. Harvey, "Cross-phase modulational instability in high-birefringence fibers," Opt. Commun. 78, 137-142 (1990).

16. E. Seve, P. Tchofo Dinda, G. Millot, M. Remoissenet, J. M. Bilbault, and M. Haelterman, "Modulational instability and critical regime in a highly birefringent fiber," Phys. Rev. A 54, 3519 (1996).

17. J. F. L. Freitas, C. J. S. de Matos, M. B. Costa e Silva, and A. S. L Gomes, "Impact of phase modulation and parametric gain on signal polarization in an anomalously dispersive optical fiber," J. Opt. Soc. Am. B 24, 1469-1474 (2007).

18. V. V. Kozlov, J. Nun̄o, and S. Wabnitz, "Theory of lossless polarization attraction in telecommunication fibers: erratum," J. Opt. Soc. Am. B 29, 153-154 (2012).

19. V. V. Kozlov and S. Wabnitz, "Suppression of relative intensity noise in fiber-optic Raman polarizers," IEEE Photon. Technol. Lett. 23, 1088-1090 (2011).

20. V. V. Kozlov, J. Nuño, J. D. Ania-Castañón, and S. Wabnitz "Multi-channel Raman polarizer with suppressed relative intensity noise for WDM transmission lines," submitted to Opt. Lett. (2012). 